\title{
A new concept of reliability system and applications in finance
}

\author{
Roy Cerqueti ${ }^{1,2}$ (D) \\ Accepted: 3 June 2021 / Published online: 8 June 2021 \\ (c) The Author(s) 2021
}

\begin{abstract}
This paper proposes and explores an extension of the usual $k$-out-of- $n$ systems, where the components of the system are assumed to play different roles in determining its failure and with not necessarily i.i.d. components lifetimes. The theoretical reliability framework is compared with the standard weighted $k$-out-of- $n$ systems, and it is actually adopted for the development of a financial derivative model whose outcome depends on the crossing of some predefined barriers of a set of assets. More precisely, such a derivative is presented as a coherent system whose components are the assets of the basket.
\end{abstract}

Keywords Reliability $\cdot k$-out-of- $n$ system $\cdot$ Finance $\cdot$ Signature of a system $\cdot$ Financial derivatives

\section{Introduction}

Reliability theory is basically grounded on the fundamental task of optimizing the lifetime of a system. In several cases, the investigated system is composed by distinct components. In such a situation, the lifetime of the system depends on the ones of its components, and the study of this context brings challenging themes in mathematical statistics and operations research.

A noticeable contribution in reliabilty theory is the monograph of Spizzichino (2001), where a detailed toolkit on stochastic dependence and ageing along with some notions on survival probabilities is provided. In this respect, the dependence structure among system's components is an important aspect to be taken into full consideration. Navarro et al. (2005)

I would like to thank Fabio Spizzichino for helpful suggestions and comments.

Roy Cerqueti

roy.cerqueti@uniroma1.it

1 Sapienza University of Rome, Department of Social and Economic Sciences, Piazzale Aldo Moro, 5, 00185 Rome, Italy

2 London South Bank University, School of Business. 103 Borough Road, London SE1 0AA, UK 
explore this topic by comparing different systems whose components are not independent. Further work on this has been carried out by Dembinska (2018), Navarro et al. (2013), Navarro et al. (2015), Parsa et al. (2018), Zhang (2021).

In the wide family of reliability systems, a prominent role is played by those whose failure depends on the pure number of the failed components. In this context, an $n$-component system that works when at least $k$ of its components work-or, in the same way, that fails when at least $k$ of its components fail-is called $k$-out-of- $n$ system. Such a type of system is widely employed in reliability theory, and has been formalized more than fifty years ago (see (Birnbaum et al. 1961; Esary and Proschan 1963), and see also the related extensive treatment of Barlow and Proschan (1981)). Their relevance for practical applications and for fostering methodological studies is witnessed by the popularity of such a theme in the recent literature (see e.g. Eryilmaz 2011, 2012, 2013; Franko and Tutuncu 2016; Freixas and Puente 2009; Gurler and Bairamov 2009; Petchrompo et al. 2020; Zhang et al. 2019).

The original definition of a $k$-out-of- $n$ system assigns an identical role to all the components in determining the failure of the overall system. Moreover, the original setting introduces also components lifetimes which are i.i.d. Such assumptions are restrictive when dealing with practical applications of this reliability instrument. This paper overcomes such limitations by presenting an extension of the $k$-out-of- $n$ systems, as we will see in details below. We are here interested in the events of failure. Such an extension will be compared also with the weighted $k$-out-of- $n$ reliability systems introduced in Wu and Chen (1994), to highligh similarities and discrepancies between them. The usefulness of the proposed novel concept of reliability systems for applied science modeling will be shown in the field of finance, with a specific reference to the theory of financial derivatives.

We enter the details.

We consider a reliability system with $n$ components. We assume that the system fails when, jointly, we have that

(i) $k$ components fail;

(ii) "some" of the failed components belong to a predefined special set with cardinality $r \in\{0,1, \ldots, n\}$.

The number of the components in such a special set which is needed for having the failure of the system depends on $k$, and will be identified through a function $\rho$ as $\rho(k)$ (see details in the next section). The resulting system will be denoted as $k / \rho(k)$-out-of- $n / r$ system.

Some variants have been proposed to let the original $k$-out-of- $n$ system be more tailored on specific reliability issues. The scientific ground of the most part of such variants lies in the need-for practical applications_-of assigning different relevance to the components of the systems when computing their reliability [see e.g. Borgonovo (2010) and (Eryilmaz 2018) for a discussion on this point]. A noticeable extension of the original setting is obtained by assuming that the system works when the elements of some special sets of connected components work (see e.g. Yamamoto et al. 2008).

In this respect and as mentioned above, particularly close to our context are the so-called weighted $k$-out-of-n systems, introduced in Wu and Chen (1994). In such models, all the components of the system are endowed with some weights $w$ 's, and the system is assumed to fail when the aggregated weight of the not failed components is below a prefixed threshold $\Theta$. Our methodological proposal departs from the original conceptualization of the weighted $k$-out-of- $n$ systems, as we will discuss in details below. However, under some conditions, a set of weights $w$ 's and a threshold $\Theta$ can be opportunely selected to identify the $r$ components introduced above and mimic the behavior of $r h o$ in the definition of the $k / \rho(k)$-out-of$n / r$ systems. Such selections are not unique, and any $k / \rho(k)$-out-of- $n / r$ system identifies 
a class of weighted $k$-out-of- $n$ systems with an infinite number of elements. Moreover, as we will see below, the properties of the $k / \rho(k)$-out-of- $n / r$ systems depend on the set of the $r$ components introduced above and on the definition of $\rho$, and do not refer in any case to a possible selection of the components weights $w$ 's and of the threshold $\Theta$. Therefore, any $k / \rho(k)$-out-of- $n / r$ system provides some general features of an infinite collection of weighted $k$-out-of- $n$ systems. We have provided a discussion on the relationship between our proposal and the weighted $k$-out-of- $n$ systems in Sect. 2.

Special attention has been also paid in our framework to the different roles played by the components of the reliability system. Specifically, the (Barlow and Proschan 1975) importance index of the components of the system has been computed, to provide a measure of the relevance of each of them. In doing so, we are in linee with the recent contributions of Borgonovo et al. (2016), Eryilmaz (2016), Gao et al. (2018), Sheng and Ke (2020) and Wu and Coolen (2013), where some further developments of the concept of important index are also presented.

In the original setting, the reliability function of a $k$-out-of- $n$ system is explored under the assumption of i.i.d. components. However, we believe that such an assumption is rather restrictive in the financial context, even if there is evidence of scientific literature dealing with sets of assets with i.i.d. returns (see e.g. Alexander and Venkatramanan 2012; Chen et al. 2015; Bayer et al. 2015 ). Thus, we will consider the case of systems with exchangeable components, which still allows to treat mathematically our model by also including a dependence structure among the assets. In particular, we mention the classical studies on exchangeability and positive dependence structure (see e.g. Shaked and Tong (1985)), pointing to the general idea of positive correlations among assets and risky portfolios. Moreover, the case of i.i.d. components is a special case of the exchangeable setting. Therefore, we employ our reliability arguments to construct a financial model for derivatives theory based on the proposed extension of the $k$-out-of- $n$ systems. We will introduce some notes on financial derivatives, along with the formalization of the financial model, in Sect. 3.

More specifically, we adopt the extension of $k$-out-of- $n$ systems mentioned above for defining a special subfamily of barrier basket options, where the basket is formed by assets whose joint distribution is exchangeable. The components of each option-system are given by the assets forming the basket. Furthermore, options are reasonably viewed here as coherent systems (see e.g. Barlow and Proschan 1981).

The reliability function of the system will be financially translated in the probability that the payoff of the option is positive at a given date (not necessarily the expiration one but, obviously, not after it). The computation of the reliability function is implemented by employing the notion of signature of the system, which has been introduced by Samaniego (1985) and extensively studied in the context of coherent systems by Kochar et al. (1999), Boland and Samaniego (2004), Samaniego (2007), Spizzichino (2008), Navarro et al. (2010), Gertsbakh and Shpungin (2010), Marichal and Mathonet (2011), Marichal et al. (2015) and references therein.

To the best of our knowledge, the concept of signature has been used so far only in the frame of reliability theory. However, such a notion may be efficiently employed also in the analysis of systems in other fields. In particular, signature can play a useful role in option theory (see Cerqueti and Spizzichino, 2014 for a very preliminary analysis).

To sum up, this paper offers some new contributions to the existing scientific debate.

Under a methodological perspective, our paper extends the standard concept of $k$-outof- $n$ systems. It does so in two main respects: by one side, we remove the restrictive equal weights assumption and cluster the components of the system into two different categories, on the basis of their relevance; by the other side, we abandon the i.i.d. condition for the 
failure times of the components and allow a dependence structure among them. Thus, our proposal overcomes the limited usefulness of the standard $k$-out-of- $n$ systems for applications purposes. Moreover, the presented novel setting is comparable-and it is actually compared in the paper-with the weighted $k$-out-of- $n$ systems in a natural way.

By a more financial point of view, we offer a new model for a special class of derivativessome types of barrier basket options — on the ground of reliability theory. In doing so- - for the first time, to the best of our knowledge - we propose an informative and original application of some relevant concepts of reliability to the area of finance.

The remaining part of the paper is organized as follows. Section 2 contains the proposed extension of the standard $k$-out-of- $n$ models and explores its reliability function by computing its signature. Such a section contais also a discussion on the comparison between our methodological proposal and the weighted $k$-out-of- $n$ systems. Section 3 is devoted to a general discussion of the financial products of interest, along with relevant literature. It contains also the formalization of the barrier basket option model, which is derived by adapting the reliability framework we deal with. Some examples are also given to assist the reader in catching the links between finance and reliability theory. Section 4 presents a discussion of the different roles played by the components of the system. Last Section offers some conclusive remarks.

\section{The $k / \rho(k)$-out-of- $n / r$ systems}

This section is devoted to the introduction of the reliability system we deal with. As we will see below, our methodological proposal can be effectively applied in the context of financial derivatives. Beyond the theoretical definition, we will present below a couple of examples in the financial environment (see Examples 2 and 3). However, we here propose a very simple general example coming from real life, to assist the reader in following the flow of the methodological arguments. In so doing, we propose also some notation that will be used in the construction of the model.

Example 1 Consider a University with an Operations Research Department, with three full professors, four associate professors and six assistant professors. Assume that-according to the regulation of the Italian Academic system - full and associate professors are in charge for 120 hours teaching every year, while assistant professor have 60 hours. The total amount of teaching hours is then 1200 hours.

Assume that each professor may change her/his status and become not affiliated to the Department. The reason for such a change of status is not of interest here. When a professor is out from the Department, she/he stops teaching.

We assume that each professor is ready to take 20 extra teaching hours, to cover the colleagues who change their status. We also assume that the Department is in financial distress, and it cannot assign extra hours to external professors.

Under the reliability context we deal with, we say that professors may be viewed as components of the system Department. A professor "fails" when she/he goes out from the Department, and the Department "fails" when it is not able to sustain the courses and the teaching hours.

In this setting, we have two different sets of professors, according to the different teaching duties. One of them-say $\mathcal{I}$-is composed by full and associate professors, while the other one-namely, $\mathcal{N}$ - contains the assistant professors. The cardinality of $\mathcal{I}$ is $r=7$, while set $\mathcal{N}$ contains $n-r=6$ professors. The distinction between such types of professors is natural, 
in that they have different teaching hour duties and their failures have different impacts on the overall system. In this respect, the elements of $\mathcal{I}$ are more relevant than the ones of $\mathcal{N}$.

If one professor fails, then the system does not fail-even if the failed professor belongs to $\mathcal{I}$. Indeed, in this case, the number of professors becomes equals to eleven, and six of them will teach 20 hours more.

If two professors fail, then professors affiliated to the Department become ten. In this case, the originating sets of the failed professors is relevant. In the unlucky case that both of the failed professors belong to in $\mathcal{I}$, then the system fails. When both professors are in $\mathcal{N}$, then the system does not fail, and six of the remaining professors will teach extra 20 hours. When one professor is in $\mathcal{N}$ and the other is in $\mathcal{I}$, then the system does not fail, and nine professors will teach 20 hours extra. To sum up, when two professors fail, then they have to be both in $\mathcal{I}$ to have the failure of the system.

If three professors in $\mathcal{N}$ fail, then each of the remaining nine professors will have in charge 20 hours extra and the system does not fail. But, if at least one of the failed professors belong to $\mathcal{I}$, then the system fails. Thus, if three professors fail, then at least one of them has to be in $\mathcal{I}$ to have the failure of the system.

If four or more than four professors fail, then the system fails, even if all them are in $\mathcal{N}$.

With Example 1 in mind, we are confident that the following formal arguments will be more convincing.

Consider a probability space $(\Omega, \mathcal{F}, \mathbb{P})$, on which all the random variables that will be introduced in this paper are defined. The expected value operator with respect to $\mathbb{P}$ will be denoted by $\mathbb{E}$.

We introduce a reliability binary system $\mathbf{S}$ whose $n$ components are denoted by $C_{1}, \ldots, C_{n}$. The set collecting the components will be denoted by $\mathcal{B}$.

The state of the system $\mathbf{S}$ at time $t>0$ is:

$$
Y(t)= \begin{cases}1 & \text { if the system has not failed in }(0, t], \\ 0 & \text { otherwise }\end{cases}
$$

while the state of the $j$-th component $C_{j}$ at time $t$ is denoted by:

$$
Y_{j}(t)= \begin{cases}1 & \text { if } C_{j} \text { has not failed in }(0, t], \\ 0 & \text { otherwise. }\end{cases}
$$

The structure function of the system is $\phi:\{0,1\}^{n} \rightarrow\{0,1\}$

$$
Y(t)=\phi\left(Y_{1}(t), \ldots, Y_{n}(t)\right),
$$

and it identifies the configurations of the components which cause the failure of the system.

Thus, the system lifetime is:

$$
\mathcal{T}:=\inf \left\{t \geq 0 \mid \phi\left(Y_{1}(t), \ldots, Y_{n}(t)\right)=0\right\} .
$$

Analogously, by (2), we can define the $n$-dimensional vector of components lifetimes as $\mathbf{X}=\left(X_{1}, \ldots, X_{n}\right)$, where

$$
X_{j}=\inf \left\{t>0 \mid Y_{j}(t)=0\right\}
$$

is the lifetime of the individual $j$-th component of the system.

For $t \geq 0$, the reliability function of the system at time $t$ is

$$
R_{S}(t) \equiv \mathbb{P}\{\mathcal{T}>t\}
$$


As intuition suggests, the stochastic model assumed for the random vector $\mathbf{X}$ plays an active role for the assessment of the quantity in (6). In reliability theory, it is often assumed that the components are i.i.d.. However, since we want to present here financial applications, we relax such a condition, and assume that the following assumption stands in force hereafter.

Assumption 1 The random vector $\mathbf{X}$ has an absolutely continuous distribution $F$ and exchangeable components.

For the convenience of the reader, we recall that $\mathbf{X}=\left(X_{1}, \ldots, X_{n}\right)$ is said to have exchangeable components when the joint probability distribution of the components $X_{1}, \ldots, X_{n}$ is the same of the one of the random variables $X_{\sigma(1)}, \ldots, X_{\sigma(n)}$, for each permutation $(\sigma(1), \ldots, \sigma(n))$ of $(1, \ldots, n)$.

We will discuss below the exchangeability assumption and focus now on the absolute continuity, which plays a key role in our context. Indeed, by including the time dimension in the problem, we notice that the system fails after some consecutive failures of its components, i.e.: in correspondence of the $k$-th failure. Thus, the assumption that $F$ is absolute continuous guarantees that

$$
\mathbb{P}\left\{X_{(1)} \neq \ldots \neq X_{(n)}\right\}=1,
$$

so that:

$$
\mathbb{P}\left\{\mathcal{T}=X_{(k)}, \text { for some } k\right\}=1,
$$

where $X_{(1)}, \ldots, X_{(n)}$ the order statistics of $\left(X_{1}, \ldots, X_{n}\right)$. Substantially, formula (7) means that the failure of the system is simultaneous to the failure of only one component.

Now, consider the partition $\left\{E_{k}\right\}_{k=1, \ldots, n}$ of the sample space $\Omega$, with

$$
E_{k} \equiv\left\{\mathcal{T}=X_{(k)}\right\}, \quad k=1, \ldots, n,
$$

so that:

$$
\mathcal{T}=\sum_{k=1}^{n} X_{(k)} \mathbf{1}_{E_{k}}
$$

Hence:

$$
R_{S}(t)=\sum_{k=1}^{n} p_{k} \cdot \mathbb{P}\left\{X_{(k)}>t \mid E_{k}\right\},
$$

where $p_{k}=\mathbb{P}\left(E_{k}\right)$, for each $k=1, \ldots, n$.

The vector $\mathbf{p}=\left(p_{1}, \ldots, p_{n}\right)$ is the signature of the system $\mathbf{S}$ (see (Samaniego 1985)), and depends on the structure function of $\mathbf{S}$. By construction, the signature provides a detailed information on the connection between the number of failed components and the failure of the overall system. Indeed, its generic component $p_{k}$ represents the probability that the system fails when the consecutive failure of $k$ components of the system is observed.

The vector $\mathbf{p}$ plays a relevant role in identifying the reliability function of the system, in the light of the exchangeability condition in Assumption 1. Indeed, when the components' lifetimes are exchangeable, then it is easy to show (see e.g. Spizzichino (2008)) that

(i) the events $\left\{X_{(k)}>t\right\}$ and $E_{k}$ are independent, for each $k=1, \ldots, n$;

(ii) one has

$$
R_{S}(t)=\sum_{k=1}^{n} p_{k} \cdot \mathbb{P}\left\{X_{(k)}>t\right\},
$$


Thus, in the exchangeable case, the signature, along with the distribution of the lifetimes of the components, provides full information on the reliability function of the system.

In the context described above, we now extend the usual $k$-out-of- $n$ models and consider the case where there exists a distinction among the components of the system.

In particular, we assume that the $n$ components can be clustered in two non-overlapping categories: $r$ components are the "important" ones while the remaining $s=n-r$ are "standard" components. The set of the former components will be denoted by $\mathcal{I}$, while $\mathcal{N}$ denotes the complementary set formed with the latter ones, so that

$$
\mathcal{I} \cap \mathcal{N} \equiv \emptyset, \quad \mathcal{I} \cup \mathcal{N} \equiv \mathcal{B} .
$$

Conventionally, we label the components in such a way that $\mathcal{I}=\left\{C_{1}, \ldots, C_{r}\right\}$. The sets $\mathcal{I}$ and $\mathcal{N}$ allow us to identify different types of reliability systems.

Specifically, define a nonincreasing function

$$
\rho:\{1, \ldots, n\} \rightarrow\{0,1, \ldots, r+1\},
$$

such that the system fails at the first time in which the failures of $k$ components are observed, with at least $\rho(k)$ failures due to the important components. Thus, $\rho$ is a function of the number $k$ of failed components and, as explained in details below, it associate to such a number a threshold $\rho(k)$ of important assets that have to fail in order to have the failure of the system. In this way, if the number of failed assets is $k$ but the number of failed important assets is lower than $\rho(k)$, the system does not fail. As we will formally see below, intuition suggests that the number of failures of important assets needed for the failure of the system decreases as the number of failed components grows. This explains the condition of $\rho$ being not increasing.

The resulting reliability model represents an actual extension of the usual $k$-out-of- $n$ systems. In fact, the proposed setting collapses in the $k$-out-of- $n$ system when $r=0$ and $\rho(k)=0$, for each $k$ or, alternatively, $r=n$ and $\rho(k)=k$, for each $k$.

Such an extended model will be called a $k / \rho(k)$-out-of- $n / r$ system.

For $\mathcal{I}$ and $\mathcal{N}$ given, different choices of the function $\rho$ give rise to different types of reliability systems.

In any case, the function $\rho$ has the following meaning:

- when $\rho(k)=0$, then the failure of $k$ components belonging to $\mathcal{N}$ is enough to determine the failure of the system;

- the position $\rho(k)=r+1$ means that $k$ is so small that the failure of $k$ components cannot produce the system's failure, even in the case when all the failed components are in $\mathcal{I}$;

- the minimum number of components' failures able to potentially cause the failure of the system is the minimum value of $k$ satisfying the condition $\rho(k) \leq k$;

- the maximum possible number of components' failures that can be conceptually observed up to the system's failure does coincide with the minimum value of $k$ such that $\rho(k)=0$.

The structure function of the system depends on the function $\rho$, and can be written as follows:

$$
\phi\left(y_{1}, \ldots, y_{n}\right)=\left\{\begin{array}{l}
0, \text { if } \rho\left(n-\sum_{j=1}^{n} y_{j}\right) \leq r-\sum_{j=1}^{r} y_{j} ; \\
1, \text { if } \rho\left(n-\sum_{j=1}^{n} y_{j}\right)>r-\sum_{j=1}^{r} y_{j} .
\end{array}\right.
$$

In fact, the system fails as soon as the following relations are simultaneously true:

$$
\left\{\begin{array}{l}
\sum_{j=1}^{n} y_{j}=n-k \\
\sum_{j=1}^{r} y_{j} \leq r-\rho(k),
\end{array}\right.
$$


namely (14) is equivalent to the first condition in (13).

We are now ready to compute the signature of a $k / \rho(k)$-out-of- $n / r$ system, so that formula (11) gives its reliability function.

Fix $k=1, \ldots, n$. It is simple to check that, for any function $\rho$, we have:

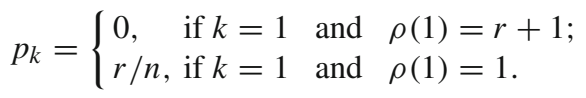

- $p_{k}=0$ if $k>1$ and $\rho(k)=r+1$;

- $p_{k}=0$ if $k>1$ and $\rho(k)=\rho(k-1)=0$;

- $p_{k}=1-\sum_{h \neq k} p_{h}$ if $k>1$ and $\rho(k)=0, \rho(k-1)>0$.

Let us consider now the case of $k=2, \ldots, n$ such that $0<\rho(k) \leq k$. We introduce

$$
\bar{s}_{k}=\mathbb{P}\left(r-\sum_{i \in \mathcal{I}} y_{i} \leq \rho(k), \sum_{i=1}^{n} y_{i}=n-k\right),
$$

where $y_{i}=0,1$ and which represents the probability of having no more than $\rho(k)$ failures of important components when $k$ components fail.

Assumption 1 guarantees that

$$
\bar{s}_{k}=\sum_{\ell=0}^{\rho(k)-1} \frac{\left(\begin{array}{l}
r \\
\ell
\end{array}\right)\left(\begin{array}{l}
n-r \\
k-\ell
\end{array}\right)}{\left(\begin{array}{l}
n \\
k
\end{array}\right)} .
$$

Therefore, the probability of exactly $\rho(k)$ failures of important components when $k$ components had failed-i.e.: the $k$-th component of the signature of the system $\mathbf{S}$-is given by:

$$
p_{k}=\bar{s}_{k-1}-\bar{s}_{k}=\sum_{\ell=0}^{\rho(k-1)-1} \frac{\left(\begin{array}{c}
r \\
\ell
\end{array}\right)\left(\begin{array}{c}
n-r \\
k-1-\ell
\end{array}\right)}{\left(\begin{array}{c}
n \\
k-1
\end{array}\right)}-\sum_{\ell=0}^{\rho(k)-1} \frac{\left(\begin{array}{l}
r \\
\ell
\end{array}\right)\left(\begin{array}{c}
n-r \\
k-\ell
\end{array}\right)}{\left(\begin{array}{l}
n \\
k
\end{array}\right)} .
$$

The different roles played by the important and standard components of the system is also highlighted by the importance index of Barlow and Proschan (1975), which assume different values when it is computed for an element of $\mathcal{I}$ or $\mathcal{N}$.

In fact, given $j \in \mathcal{B}$, the Barlow-Proschan importance index for $j$ represents the probability of having the failure of the system when the status of $j$ moves from 1 to 0 . In our context, it is given by:

$$
\gamma_{j}=\frac{1}{n} \sum_{k=0}^{n-1} \frac{1}{\left(\begin{array}{c}
n-1 \\
k
\end{array}\right)} \sum_{A \subset\{1, \ldots, n\} \backslash\{j\}:|A|=k}[\phi(A \cup\{j\})-\phi(A)],
$$

where $\phi(A)=\phi\left(y_{1}, \ldots, y_{n}\right)$ with $y_{h}=\mathbf{1}_{\{h \in A\}}$, for each $h=1, \ldots, n$.

The generic set $A \in\{1, \ldots, n\}$ contributes to $\gamma_{j}$ in (18) only when $\phi(A)=0$ and $\phi(A \cup\{j\})=1$.

Now, assume that $A=\mathcal{I}_{A} \cup \mathcal{N}_{A}$, where $\mathcal{I}_{A} \subset \mathcal{I}, \mathcal{N}_{A} \subset \mathcal{N}$ and $\left|\mathcal{I}_{A}\right|+\left|\mathcal{N}_{A}\right|=k$.

The term $\gamma_{j}$ in formula (18) depends on the nature of $j$, which can be of standard or important type. Two cases can be distinguished:

Case $\mathcal{I}$ if $j \in \mathcal{I}$, then $\phi(A)=0$ if and only if $r-\left|\mathcal{I}_{A}\right| \geq \rho(n-k)$, while $\phi(A \cup\{j\})=1$ if and only if $r-\left|\mathcal{I}_{A}\right|-1<\rho(n-k-1)$. To sum up:

$$
\rho(n-k) \leq r-\left|\mathcal{I}_{A}\right|<\rho(n-k-1)+1,
$$


which leads to $\left|\mathcal{I}_{A}\right|>r-\rho(n-k-1)-1$.

Case $\mathcal{N}$ if $j \in \mathcal{N}$, then $\phi(A)=0$ if and only if $r-\left|\mathcal{I}_{A}\right| \geq \rho(n-k)$, while $\phi(A \cup\{j\})=1$ if and only if $r-\left|\mathcal{I}_{A}\right|<\rho(n-k-1)$. To sum up:

$$
\rho(n-k) \leq r-\left|\mathcal{I}_{A}\right|<\rho(n-k-1),
$$

specifically leading to $\left|\mathcal{I}_{A}\right|>r-\rho(n-k-1)$.

Therefore, by (18) and by Case $\mathcal{I}$ and Case $\mathcal{N}$ listed above, one has

$$
\gamma_{j}=\left\{\begin{array}{l}
\frac{1}{n} \sum_{k=0}^{n-1} \frac{1}{\left(\begin{array}{c}
n-1 \\
k
\end{array}\right)} \mid\{A \subset\{1, \ldots, n\} \backslash\{j\}:|A| \\
\left.=k, \rho(n-k) \leq r-\left|\mathcal{I}_{A}\right|<\rho(n-k-1)+1\right\} \mid, j \in \mathcal{I} ; \\
\frac{1}{n} \sum_{k=0}^{n-1} \frac{1}{\left(\begin{array}{c}
n-1 \\
k
\end{array}\right)} \mid\{A \subset\{1, \ldots, n\} \backslash\{j\}:|A| \\
\left.=k, \rho(n-k) \leq r-\left|\mathcal{I}_{A}\right|<\rho(n-k-1)\right\} \mid \quad j \in \mathcal{N} .
\end{array}\right.
$$

As expected, formula (19) gives that $\gamma_{j_{I}}>\gamma_{j_{N}}$, for $j_{I} \in \mathcal{I}$ and $j_{N} \in \mathcal{N}$.

\subsection{Relationship between the $k / \rho(k)$-out-of- $n / r$ systems and standard $k$-out-of-n weighted ones}

We now present some arguments on the comparison between the $k / \rho(k)$-out-of- $n / r$ systems and the classical $k$-out-of- $n$ weighted ones.

First of all, we recall that a $k$-out-of- $n$ weighted system is a reliability bynary $k$-out-of- $n$ system $\mathbf{S}_{w}$ with $n$ components $C_{1}^{w}, \ldots, C_{n}^{w}$ such that the $j$-th component $C_{j}^{w}$ is endowed with a weight $w_{j}>0$, for each $j=1, \ldots, n$. Once the $j$-th component fails, then it disappears from the set collecting the active components and its weight becomes null. Thus, the value of a given weight might change over time, since it can pass from a positive value to zero. We denote the $j$-th weight at time $t$ by $w_{j}(t)$. Also in this case, we assume that the system is coherent, so that at time $t=0$ all the weights $w_{1}(0), \ldots, w_{n}(0) \multimap$ or, briefly and as introduced above, $w_{1}, \ldots, w_{n}$-are positive. The system is failed at time $t$ when the sum of the weights of the active components at time $t$ is less than or equal to a prefixed threshold $\Theta>0$, i.e. when

$$
\sum_{j=1}^{n} w_{j}(t) \leq \Theta .
$$

We now show that some classes of the $k$-out-of- $n$ weighted systems described above can be seen as $k / \rho(k)$-out-of- $n / r$ systems by properly selecting function $\rho$ and the sets $\mathcal{I}$ and $\mathcal{N}$.

Without loss of generality, let us assume that the indices of the components are taken in order to have $w_{1}<\cdots<w_{n}$.

Fix a threshold $\Theta>0$ as in condition (20). There exists an integer $n_{\Theta} \in\{0,1, \ldots, n\}$ such that

$$
\sum_{j=1}^{n_{\Theta}} w_{j} \leq \Theta \quad \text { and } \quad \sum_{j=1}^{n_{\Theta}+1} w_{j}>\Theta,
$$

with the conventional agreement that

$$
\sum_{j=1}^{0} w_{j}=0
$$


The index $n_{\Theta}$ represents a threshold which captures the minimum number of components that have to fail to see surely the failure of the system. Indeed, if one observes $n_{\Theta}+1$ consecutive failures of the components, then the system is surely failed. If one has $n_{\Theta}$ failed components, then one might have still a working system in the case in which the failed components are $C_{1}^{w}, \ldots, C_{n_{\Theta}}^{w}$. The corner case $n_{\Theta}=0$ captures the trivial situation in which condition (20) cannot be verified and the system cannot fail, even if all its components fail. It is included here for completeness, but under the considered hypothesis of coherent system one has $n_{\Theta}>0$. Analogously, the corner case of $n_{\Theta}=n$ is associated to the trivial situation in which condition (20) is always verified, so that the system is already failed at time $t=0$, when all its components are active. Also this situation is excluded by the hypothesis of coherence, and it is here reported for the sake of completeness. To remove the corner cases, which then violate the assumption on the coherence of the system, the weights $w$ 's and the $\Theta$ have to be not trivial. As an example, corner cases are removed in the natural situation of $w_{1}<\Theta<w_{n}$.

Under some conditions on the weights, we can translate the framework described above as a peculiar $k / \rho(k)$-out-of- $n / r$ system.

We consider the collection of standard components $\mathcal{N}$ and important ones $\mathcal{I}$ intuitively as follows:

$$
\mathcal{N}=\left\{C_{1}^{w}, \ldots, C_{n_{\Theta}}^{w}\right\}, \quad \mathcal{I}=\left\{C_{n_{\Theta}+1}^{w}, \ldots, C_{n}^{w}\right\} .
$$

In this case, the cardinality of the set $\mathcal{I}$ is $r=n-n_{\Theta}$.

Function $\rho$ can be defined in presence of some conditions on the weights of the components and on the selected value of $\Theta$, and according to the definition of the sets $\mathcal{N}$ and $\mathcal{I}$ in (21). The following proposition formalizes such requirements, and gives also the related expression of function $\rho$.

Proposition 1(r1) The following conditions are equivalent:

- one has

$$
\sum_{j=1}^{n-h} w_{j}>\Theta, \quad \text { with } h \in\left\{0, \ldots, n-n_{\Theta}-1\right\}
$$

- $\rho(k)=r+1$, for each $k=1, \ldots, h$.

(r2) The following conditions are equivalent:

- for $k \in\{1, \ldots, n\}$ and $s \in\{1, \ldots, k\}$, one has

$$
\left\{\begin{array}{l}
\sum_{j=k-s+1}^{n} w_{j}-\sum_{\ell=1}^{s} w_{n \Theta+\ell} \leq \Theta, \\
\sum_{j=1}^{n-s+1} w_{j}-\sum_{\ell=0}^{k-s} w_{n_{\Theta}-\ell}>\Theta
\end{array}\right.
$$

$-\rho(k)=s$.

(r3) The following conditions are equivalent:

- for $h \in\left\{n_{\Theta}, \ldots, n-1\right\}$

$$
\sum_{j=h+1}^{n} w_{j} \leq \Theta
$$

- $\rho(k)=0$, for each $k=1, \ldots, h$. 
The proof of Proposition 1 can be obtained by straightforward computation, and it is omitted.

Proposition 1 provides some necessary and sufficient conditions for correctly identifying function $\rho$. It is important to notice that conditions (22), (23) and (24) are not satisfied in all the $k$-out-of- $n$ weighted systems, and identify a class of such systems which can be viewed as $k / \rho(k)$-out-of- $n / r$ ones. As a consequence, not all the $k$-out-of- $n$ weighted systems can be translated into $k / \rho(k)$-out-of- $n / r$ ones.

However, by a different perspective, our proposal exhibits an interesting aspect of qualitative universality. More specifically, each $k / \rho(k)$-out-of- $n / r$ system identifies an infinite number of $k$-out-of- $n$ weighted systems. In fact, once the cardinalities of the sets $\mathcal{N}$ and $\mathcal{I}$ are given and function $\rho$ is defined, there is an infinite set of weights $w$ 's and infinite thresholds $\Theta$ such that conditions (22), (23) and (24) are satisfied.

\section{Financial application: a barrier basket option model}

This section is devoted to the development of an option model in the light of the $k / \rho(k)$-outof- $n / r$ systems introduced above. When possible, we maintain the same notation adopted in the previous section.

We preliminarly provide a description of the financial context we deal with, along with some relevant literature contributions.

\subsection{Overview on financial derivatives and relevant literature}

Derivatives represents one of the most studied topics in finance. Since the beginning of option pricing formalization with the celebrated paper of Black and Scholes (1973), a long history has been developed by academicians as well as by practitioners. Derivatives have been classified into several families, starting from the simplest contracts to products with a high level of complexity. For a survey on derivative and option theory, we refer to the classical monograph by Hull (2006) and, more recently, to McNeil et al. (2015).

Among all the existing typologies of derivatives, we will concentrate attention on the ones whose evolution depends on a basket of assets and on how their prices cross some prefixed barriers. Such derivatives are experiencing an increasing popularity among the retail investors in view of their constitutive features. Indeed, the basket of the assets and the related barriers can be properly selected by the investors on the basis of their risk-profiles and returns.

We notice that the considered derivative share some peculiar constitutive characteristics of the barrier basket options (see Hull 2006). Indeed, also barrier basket options value depends on whether the portfolio in the basket will cross a prefixed barrier at or within the expiration date. Therefore, we will include the considered derivative in the set of the barrier basket options and we will refer to such a category of options hereafter.

The payoff of a basket option - and, consequently, the price of it - is then strongly dependent on the composition of the basket, as an endless literature on the field clearly states (see e.g. Hobson et al. 2005; Abrahams et al. 2006 and, more recently, Sesana et al. 2014).

Barrier basket options represent a subclass of the general basket options. In this case, the payoff of the option is linked to the crossing of prefixed thresholds by the returns of the assets in the basket. For an overview of the barrier basket options and of their properties, see the monograph by Zhang (1997) but also e.g. Brockman and Turtle (2003) and, more recently, Peña et al. (2012) and Kim et al. (2015). Once the basket is fixed, the selection of the thresholds leads to the identification of the risk profile and of the expected return of the 
corresponding option. Thus, the mechanism of thresholds-selection contributes to control the riskiness of the option.

\subsection{Formalization of the financial model}

Think of a financial market that contains $n$ assets whose returns at time $t$ are denoted by $\Lambda_{1}(t), \ldots, \Lambda_{n}(t)$, at each $t \in[0,+\infty)$.

Our interest focuses on a special barrier basket option written on these assets and defined as follows:

The holder can exercise the option only at the expiration date $T$. The return of the option at time $T$, denoted by $\Pi(T)$, is positive if and only if "some" of the $n$ assets have maintained their own returns greater than a predefined positive barrier (knockout option) in the whole period $(0, T]$. In this case, the return of the option at time $T$ is given by the average of the returns of such assets at time $T$. If the return of the option is not positive at time $T$, then the option is said to be failed.

The barrier is denoted by $\alpha \in(0,+\infty)$.

By construction, it is self-evident that the failure of the option can occur before the expiration date $T$. In fact, if at a given time $t<T$ the returns of "some" of the assets are below the barriers $\alpha$ 's, then the option has failed, since the return of the option cannot be positive at time $T$. In this case, we say that the option has failed in the time period $(0, t]$.

The meaning of the term "some" will be clarified below and, as we will see, it involves the definition of the function $\rho$ in (12).

The barrier basket option is modeled here as a reliability system $\mathbf{S}$ whose $n$ components $C_{1}, \ldots, C_{n}$ are the assets in the basket $\mathcal{B}$.

The state of the option at time $t>0$ is $Y(t)$ as in (1), and it depends on the returns of the assets of the basket at $t$. Actually, such returns can be above or below the barrier $\alpha$. In particular, for each $t \in(0,+\infty)$ and $j=1, \ldots, n$, the comparison between the barrier $\alpha_{j}$ and the return $\Lambda_{j}(t)$ determines the state of the $j$-th component $C_{j}$ at time $t$, so that we define the state of the $j$-th asset at time $t>0$ as the binary variable

$$
Y_{j}(t)= \begin{cases}1 & \text { if } \Lambda_{j}(t)>\alpha, \\ 0 & \text { otherwise }\end{cases}
$$

The structure function $\phi:\{0,1\}^{n} \rightarrow\{0,1\}$ in (3) formally describes the dependence between the state of the option and the ones of its components. It is reasonable to require that $\phi$ is such that the option $\mathbf{S}$ is a coherent system, i.e.:

(i) $\phi(0, \ldots, 0)=0$ and $\phi(1, \ldots, 1)=1$. This condition means that when all the assets have returns not above the threshold $\alpha$, then the payoff of the option is not positive; differently, when the $j$-th return is greater than $\alpha$, for each $j=1, \ldots, n$, then the option has positive payoff.

(ii) $\phi$ is non-decreasing with respect to its components. This requirement expresses that the failure of one of the assets of the basket cannot lead to an improvement of the state of the option.

(iii) Each component of the system is "relevant". This assumption means that each component is able to determine the failure of the entire system in at least one situation. Formally, we can write that it does not exist $j=1, \ldots, n$ such that

$$
\phi\left(y_{1}, \ldots, y_{j-1}, 0, y_{j+1}, \ldots, y_{n}\right)=\phi\left(y_{1}, \ldots, y_{j-1}, 1, y_{j+1}, \ldots, y_{n}\right),
$$




$$
\text { for each }\left(y_{1}, \ldots, y_{j-1}, y_{j+1}, \ldots, y_{n}\right) \in\{0,1\}^{n-1} .
$$

It is worth noting that, by construction, the asset $C_{j}(1 \leq j \leq n)$ is capable in principle to survive at any $t$, even if $\mathbf{S}$ has already failed in $(0, t]$. According to formulas (4) and (5), the lifetimes of the option and of the $j$-th asset will be denoted by $\mathcal{T}$ and $X_{j}$, respectively, for each $j=1, \ldots, n$.

Clearly, Assumption 1 is true also for this collection of $X$ 's.

For the convenience of the reader, we now present an illustrative example.

Example 2 Consider $n=5$ assets $C_{1}, \ldots, C_{5}$, a positive threshold (barrier) $\alpha$, and suppose that the barrier basket option fails in one of the following three cases:

(c1) In the period $(0, T]$, the returns of the assets $C_{1}, C_{3}$ and $C_{4}$ go below $\alpha$;

(c2) In the period $(0, T]$, the returns of the assets $C_{2}$ and $C_{5}$ go below the threshold $\alpha$.

The structure function can be constructed on the basis of the expiration conditions $(c 1)$ and (c2) as follows:

$$
Y(T)=\phi\left(Y_{1}(T), \ldots, Y_{5}(T)\right)=\left\{\begin{aligned}
0, & \text { if }\left(Y_{1}(T), Y_{3}(T), Y_{4}(T)\right)=(0,0,0) \text { or } \\
& \left(Y_{2}(T), Y_{5}(T)\right)=(0,0) \\
1, & \text { otherwise }
\end{aligned}\right.
$$

It is easy to check that the barrier basket option in this example-with the structure function $\phi$ defined as in (26) - is a coherent reliability system.

Example 2 sheds some light also on the meaning of the term "some" in the definition of the barrier basket option. It provides a quick financial interpretation of the structure function. Of course, it is not grounded on empirical instances, even if it can be easily reproduced in the case of availability of a real financial definition of a barrier basket option.

The failure of the option as a reliability system is, indeed, caused by the joint failure of some specific set of components over the period $(0, T]$.

In particular, we assume that the barrier basket option is a $k / \rho(k)$-out-of- $n / r$ reliability system. Specifically, the basket $\mathcal{B}$ is clustered into a set of important assets $\mathcal{I}=\left\{C_{1}, \ldots, C_{r}\right\}$ and the set of standard ones $\mathcal{N}=\left\{C_{r+1}, \ldots, C_{n}\right\}$. The failure of the option is then explained by the identification of a function $\rho$ as in (12).

We now demonstrate the financial meaning of the function $\rho$ through an illustrative example.

Example 3 Consider $n=20$ assets $C_{1}, \ldots, C_{20}$, and a positive reference threshold (barrier) $\alpha$. Suppose that the barrier basket option fails in the following cases:

(d1) the returns of five assets - including at least one among $C_{1}, C_{2}, C_{3}$ and $C_{4}-$ go below the reference threshold in the period $(0, T]$;

(d2) the returns of four assets-including at least two among $C_{1}, C_{2}, C_{3}$ and $C_{4}$ - go below $\alpha$ in the period $(0, T]$;

(d3) the returns of three assets selected among $C_{1}, C_{2}, C_{3}$ and $C_{4}$ go below the reference threshold in the period $(0, T]$;

(d4) more than five assets have return going below threshold $\alpha$ in $(0, T]$.

Thus, it is easy to identify $r=4$ important assets, namely $\mathcal{I} \equiv\left\{C_{1}, C_{2}, C_{3}, C_{4}\right\}$. The remaining $n-r=16$ assets are standard ones. 
The conditions $(d 1)-(d 4)$ lead us to define the function $\rho$ as follows:

$$
\rho(k)= \begin{cases}0, & \text { if } k \geq 6 ; \\ 1, & \text { if } k=5 ; \\ 2, & \text { if } k=4 ; \\ 3, & \text { if } k=3 ; \\ 5, & \text { if } k<3\end{cases}
$$

The structure function can be constructed in a natural way on the basis of the function $\rho$, according to (13) and (27).

Example 3 traces the route for a clear understanding of the way to compute function $\rho$. As already stated above for Example 2, also in this case we can reproduce the toy framework of Example 3 in a real empirical context, once data are available.

In the specific framework we are considering, the function $R_{S}$ in (6) provides an appropriate measure of the riskiness of the option. In particular, it is important to consider the value of the function $R_{S}$ at the expiration date $T$, i.e. $R_{S}(T)$. In fact, the financial contract we deal with considers the situation of the assets' returns at the expiration date in order to establish the final payoff of the option. Thus, a correct evaluation of $R_{S}(T)$ is a crucial step for the determination of the risk profile associated to the option $\mathbf{S}$.

\section{A discussion on standard and important assets}

The choice of the sets $\mathcal{I}$ and $\mathcal{N}$ plays a key role in determining the returns of such financial products. This Section is devoted to the illustration of the different roles played by them in the final payoff of the option.

We now need the following:

Notation 1 Consider two barrier basket options $\mathbf{S}_{1}$ and $\mathbf{S}_{2}$ whose returns at time $T$ are $\Pi_{1}(T)$ and $\Pi_{2}(T)$, respectively. For $j=1,2, \mathbf{S}_{j}$ has basket $\mathbf{B}_{j}$, set of important assets $\mathcal{I}_{j}$ and set of standard ones $\mathcal{N}_{j}$. Accordingly, the function $\rho$ of the option $j$ will be denoted by $\rho_{j}$.

The $k$-th asset lifetime of option $\mathbf{S}_{j}$ will be denoted by $X_{k}^{(j)}$, for $k=1, \ldots, n$ and $j=1,2$.

The generic $\bullet$-th element of $\mathbf{B}_{j}$ will be denoted by $C_{\bullet}^{(j)}$, whose return at time $T$ is $\Lambda_{\bullet}^{(j)}(T)$, for $j=1,2$.

Furthermore, we state the following set of assumptions:

Assumption 2 The baskets $\mathcal{B}_{1}$ and $\mathcal{B}_{2}$ are disjoint sets with the same cardinality $n$, namely $\left|\mathcal{B}_{1}\right|=\left|\mathcal{B}_{2}\right|=n$.

The assets' lifetimes $X_{1}^{(1)}, \ldots, X_{n}^{(1)}$ and $X_{1}^{(2)}, \ldots, X_{n}^{(2)}$ are exchangeable.

The sets $\mathcal{I}_{1}$ and $\mathcal{I}_{2}$ share the same cardinality $r$, namely $\left|\mathcal{I}_{1}\right|=\left|\mathcal{I}_{2}\right|=r$.

Finally, $\rho_{1} \equiv \rho_{2}$, and we set $\rho=\rho_{1}=\rho_{2}$.

We now state two results which mark the difference between standard and important assets, once they are compared.

Proposition 2 Consider two barrier basket options $\mathbf{S}_{1}$ and $\mathbf{S}_{2}$. Suppose that Assumption 2 is satisfied. Moreover, suppose that:

(H1.1) For any pair $\left(C^{(1)}, C^{(2)}\right) \in \mathcal{I}_{1} \times \mathcal{I}_{2}$, one has $\mathbb{P}\left(\Lambda^{(1)}(T)>\Lambda^{(2)}(T)\right)=1$. 
(H1.2) For all $\left(C^{(1)}, C^{(2)}\right) \in \mathcal{N}_{1} \times \mathcal{N}_{2}$, one has $\mathbb{P}\left(\Lambda^{(1)}(T)=\Lambda^{(2)}(T)\right)=1$.

(H1.3) $\rho(k)>0$, for each $k=1, \ldots, n$, and there exists $\bar{k} \in\{1, \ldots, n\}$ such that $\rho(\bar{k})=\bar{k}$ and $\rho(\bar{k}-1)=r+1$.

Suppose also that the options share the same number $k_{r}$ of failed important assets and $k_{n}$ of standard ones in $(0, T]$, and the failures are such that the options have not failed in $(0, T]$.

Then $\mathbb{P}\left(\Pi_{1}(T)>\Pi_{2}(T)\right)=1$.

Proof First of all, we notice that Assumption 2 gives that also the sets of the standard assets, denoted by $\mathcal{N}_{1}$ for $\mathbf{S}_{1}$ and $\mathcal{N}_{2}$ for $\mathbf{S}_{2}$, have the same cardinality: $\left|\mathcal{N}_{1}\right|=\left|\mathcal{N}_{2}\right|=n-r$.

Second of all, hypothesis ( H1.3) and the condition of no failure of the options guarantees that $k \geq \bar{k}$, where $k=k_{r}+k_{n}$.

Now, fix $j=1,2$ and consider the barrier basket option $\mathbf{S}_{j}$. Denote by $\mathcal{O}_{\mathcal{I}_{j}} \subseteq \mathcal{I}_{j}$ and $\mathcal{O}_{\mathcal{N}_{j}} \subseteq \mathcal{N}_{j}$ the sets of the not failed assets in $(0, T]$ of important and standard type, respectively.

By hypothesis, the return of the $j$-th option at time $T$ is positive, and it is given by

$$
\Pi_{j}(T)=\frac{1}{k}\left[\sum_{i \in \mathcal{O}_{\mathcal{I}_{j}}} \Lambda^{(i)}(T)+\sum_{h \in \mathcal{O}_{\mathcal{N}_{j}}} \Lambda^{(h)}(T)\right] .
$$

The possible realizations of the return of the $j$-th option at time $T$ are given by the possible combinations of the not failed assets. Therefore, we need to define the set collecting the possible selections of $x$ elements in a set of $y$ elements, with $x$ and $y$ being integers. We denote this set by $\mathcal{Q}_{x}^{(y)}$ and, of course, $\left|\mathcal{Q}_{x}^{(y)}\right|=\left(\begin{array}{l}x \\ y\end{array}\right)$.

So, define the set

$$
\mathcal{A}\left(k, k_{r}, k_{n}\right)=\mathcal{Q}_{k_{r}}^{(r)} \times \mathcal{Q}_{k_{n}}^{(n-r)},
$$

for $k \in\{\bar{k}, \ldots, n\}, k_{r} \in\{\rho(k), \ldots, r\}$ and $k_{n}=k-k_{r}$. Then

$$
\Pi_{j}(T) \in \bigcup_{k, k_{r}, k_{n}} \bigcup_{a \in \mathcal{A}\left(k, k_{r}, k_{n}\right)}\left\{\Pi_{j}^{(a)}(T)\right\}
$$

where

$$
\Pi_{j}^{(a)}(T)=\frac{1}{k}\left[\sum_{i \in\left\{i_{1}, \ldots, i_{k_{r}}\right\}} \Lambda_{i}^{(j)}(T)+\sum_{h \in\left\{h_{1}, \ldots, h_{k_{n}}\right\}} \Lambda_{h}^{(j)}(T)\right]
$$

for each $a=\left(\left\{i_{1}, \ldots, i_{k_{r}}\right\},\left\{h_{1}, \ldots, h_{k_{n}}\right\}\right) \in \mathcal{A}\left(k, k_{r}, k_{n}\right)$.

Assumptions (H1.1), (H1.2) and (H1.3) and formula (28) assure that

$$
\mathbb{P}\left(\Pi_{1}^{\left(a_{1}\right)}(T)>\Pi_{2}^{\left(a_{2}\right)}(T)\right)=1,
$$

for each $a_{1}, a_{2} \in \mathcal{A}\left(k, k_{r}, k_{n}\right)$, for each $k, k_{r}, k_{n}$.

Now define the event

$$
\Gamma_{a}^{(j)}=\left\{C_{i}^{(j)}, C_{h}^{(j)} \text { have not failed in }(0, T] \text { iff }(i, h) \in \mathcal{A}\left(k, k_{r}, k_{n}\right)\right\}, \quad j=1,2 .
$$

By the Total Probability Theorem one has 


$$
\mathbb{P}\left(\Pi_{1}(T)>\Pi_{2}(T)\right)=\mathbb{P}\left(\bigcap_{k, k_{r}, k_{n}} \bigcap_{a_{1}, a_{2} \in \mathcal{A}\left(k, k_{r}, k_{n}\right)}\left\{\Pi_{1}^{\left(a_{1}\right)}(T)>\Pi_{2}^{\left(a_{2}\right)}(T)\right\} \cap \Gamma_{a_{1}}^{(1)} \cap \Gamma_{a_{2}}^{(2)}\right)=1,
$$

and this concludes the proof.

We now invert the role of standard and important assets as in Proposition 2, to give a distinction between the two typologies of components of the basket of an option.

Proposition 3 Consider two barrier basket options $\mathbf{S}_{1}$ and $\mathbf{S}_{2}$. Suppose that Assumption 2 is satisfied. Moreover, suppose that:

(H2.1) For any pair $\left(C^{(1)}, C^{(2)}\right) \in \mathcal{I}_{1} \times \mathcal{I}_{2}$, one has $\mathbb{P}\left(\Lambda^{(1)}(T)=\Lambda^{(2)}(T)\right)=1$.

(H2.2) For all $\left(C^{(1)}, C^{(2)}\right) \in \mathcal{N}_{1} \times \mathcal{N}_{2}$, one has $\mathbb{P}\left(\Lambda^{(1)}(T)>\Lambda^{(2)}(T)\right)=1$.

(H1.3) $\rho(k)>0$, for each $k=1, \ldots, n$, and there exists $\bar{k} \in\{1, \ldots, n\}$ such that $\rho(\bar{k})=\bar{k}$ and $\rho(\bar{k}-1)=r+1$.

Suppose also that the options share the same number $k_{r}$ of failed important assets and $k_{n}$ of standard ones in $(0, T]$, and the failures are such that the options have not failed in $(0, T]$.

Then $\mathbb{P}\left(\Pi_{1}(T)>\Pi_{2}(T)\right)<1$.

Proof We adapt here some of the parts of the proof of Proposition 2 and, when possible, the notation.

Consider $k=\bar{k}$ and $k_{r}=\bar{k}, k_{n}=0$. Then $\mathcal{O}_{\mathcal{N}_{j}}=\emptyset$, and in this specific case

$$
\Pi_{j}(T)=\frac{1}{\bar{k}}\left[\sum_{i \in \mathcal{O}_{\mathcal{I}_{j}}} \Lambda^{(i)}(T)\right] .
$$

Hypothesis (H2.1) and (31) guarantee that

$$
\mathbb{P}\left(\Pi_{1}(T)=\Pi_{2}(T)\right)>0,
$$

hence giving the thesis.

Propositions 2 and 3 provide a formal view of the different roles played by the standard and important assets in determining the failure of the option and its final return. At this aim, a suitable selection of function $\rho$-as in hypothesis $(H 1.3)$ - is needed.

In fact, as already explained above, $\rho(k)=0$ implies that the failure of $k$ assets-taken indifferently from the important and the standard ones - is enough to determine the failure of the option. Thus, in this case, one cannot appreciate the difference between standard and important assets: all of them play an identical role in determining the failure of the option.

Furthermore, the existence of $\bar{k}$ such that $\rho(\bar{k})=\bar{k}$ and $\rho(\bar{k}-1)=r+1$ assures that -at least in some cases - the failure of all important assets is required to have the failure of the option. If such a condition is violated, then $\rho(k) \neq k$ for each $k=1, \ldots, n$. In this case, we are in the position of defining a new function

$$
\tilde{\rho}:\{1, \ldots, n\} \rightarrow\{1, \ldots, n-r+1\},
$$

such that the option fails at the first time in which the failures of $k$ assets are observed, with no more than $\tilde{\rho}(k)$ failures due to the standard assets. The position $\tilde{\rho}(k)=n-r+1$ means that $k$ failures are not enough to determine the failure of the option, so that

$$
\rho(k)=r+1 \quad \text { iff } \quad \tilde{\rho}(k)=n-r+1 .
$$


Otherwise, $\rho(k)+\tilde{\rho}(k)=k$, for each $k=1, \ldots, n$, and $\tilde{\rho}(k)>0$. In this case, the thesis of Proposition 3 might be no longer true. In fact, it is trivially easy to produce examples such that the positive final return of the option is due also to some standard assets, hence invalidating the expression in (31). Therefore, (32) is not longer true.

As a consequence, the role of standard and important assets can be well illustrated only by assuming hypothesis $(H 1.3)$. In this respect, a side result of Propositions 2 and 3 is also the interpretation of function $\rho$.

\section{Conclusions and future research}

This paper contains an extension of the classical $k$-out-of- $n$ systems, obtained by assigning different roles to the components of a coherent system in term of the reliability. In particular, the components are clustered in important and standard ones, and the failure of the system depends on how many components of the two sets are failed.

The reliability framework is adapted to the construction of a barrier basket option model, so that the assets of the basket are classified in standard and important ones. We extend the standard conceptualization of the $k$-out-of- $n$ systems by considering also that assets' returns and lifetimes are exchangeable. Such a condition leads to a not restrictive setting, in that it allows to deal with assets with a stochastic dependence structure. Moreover, it includes the i.i.d. returns and lifetimes condition as a special case and has also the advantage of letting the model be tractable under a mathematical point of view. The risk profile of the option is explored by the direct computation of the reliability function of the system. Moreover, the different roles of important and standard assets are illustrated and commented.

The present study has some limitations, which might be the argument of a future exploration of such a very relevant theme.

First of all, we discuss the exchangeability condition on components lifetimes. This condition appears to be rather general, since it allows for stochastic dependence among the assets of the basket. However, it would be challenging to further extend the $k$-out-of- $n$ models under a more general assumption on the stochastic dependence, by removing the exchangeable condition for the components of the systems. In this respect, the computation of the signature and the resulting financial model offers a not easy treatment, since the equivalence between formulas (10) and (11) is not longer true.

Second, as explained above, the $k / \rho(k)$-out-of- $n / r$ systems and the weighed $k$-out-of- $n$ ones are different concepts which overlap under some conditions. In this respect, it would be interesting to discuss the financial meaning of the theoretical hypotheses leading to such an overlap. Specifically, we find particularly challenging the theme of the comparison of the $k / \rho(k)$-out-of- $n / r$ systems and the weighed $k$-out-of- $n$ ones in the context of suitably describing a barrier basket option. In the former case, we conceptualize options whose basket is compounded by two qualitative subsets of standard and important assets. The elements of such subsets are assumed to play an identical role within the reference class for the failure of the system; in the latter case-and when the weighed $k$-out-of- $n$ system cannot be seen as a $k / \rho(k)$-out-of- $n / r$ system-the assets of the basket cannot be in general clustered into two relevance-based groups, and exhibit specific individual roles for generating the failure of the system. The resulting barrier basket options are, in general, simply different, and obey different rules.

Third, this paper presents the case of clustering the system components in two setsstandard and important ones. The general case of assigning a rank to more than two subsets 
of component in terms of the importance-hence, defining different levels of importancewould be more general than our approach, and might also turn to be useful for modeling purposes.

Fourth, it is totally out of the scope of the present paper to provide a way to identify standard and important assets, once an empirical financial dataset is available. Rather than this, we discuss the mathematical difference — in terms of reliability_-between standard and important assets; we also discuss the role of standard and important assets in determining the failure of the option. However, a suitable way to financially identify standard and important assets can be a challenging exploration.

All these topics are left for future research.

Funding Open access funding provided by Università degli Studi di Roma La Sapienza within the CRUICARE Agreement.

Open Access This article is licensed under a Creative Commons Attribution 4.0 International License, which permits use, sharing, adaptation, distribution and reproduction in any medium or format, as long as you give appropriate credit to the original author(s) and the source, provide a link to the Creative Commons licence, and indicate if changes were made. The images or other third party material in this article are included in the article's Creative Commons licence, unless indicated otherwise in a credit line to the material. If material is not included in the article's Creative Commons licence and your intended use is not permitted by statutory regulation or exceeds the permitted use, you will need to obtain permission directly from the copyright holder. To view a copy of this licence, visit http://creativecommons.org/licenses/by/4.0/.

\section{References}

Abrahams, D. I., Fusai, G., \& Sgarra, C. (2006). An exact analytical solution for discrete barrier options. Finance and Stochastics, 10(1), 1-26.

Alexander, C., \& Venkatramanan, A. (2012). Analytic approximations for multi-asset option pricing. Mathematical Finance, 22(4), 667-689.

Barlow, R. E., \& Proschan, F. (1975). Importance of system components and fault tree events. Stochastic Processes and their Applications, 3, 153-173.

Barlow, R. E., \& Proschan, F. (1981). Statistical theory of reliability and life testing. Silver Spring, MD: To Begin With.

Bayer, C., Friz, P.K., \& Laurence, P., On the Probability Density Function of Baskets. In: Large Deviations and Asymptotic Methods in Finance, Eds.: Friz, P.K., Gatheral, J., Gulisashvili, A., Jacquier, A., Teichmann, J., Springer International Publishing, 449-472, 2015.

Birnbaum, Z. W., Esary, J. D., \& Saunders, S. C. (1961). Multi-component systems and structures and their reliability. Technometrics, 3(1), 55-77.

Black, F., \& Scholes, M. (1973). The pricing of options and corporate liabilities. Journal of Political Economy, $81,637-654$.

Boland, P. J., \& Samaniego, F. J. (2004). The signature of a coherent system and its applications in reliability. In T. Mazzucchi, N. Singpurwalla, \& R. Soyer (Eds.), Mathematical reliability: An expository perspective (pp. 3-30). Boston, MA: Kluwer Acad. Publ.

Borgonovo, E. (2010). The reliability importance of components and prime implicants in coherent and noncoherent systems including total-order interactions. European Journal of Operational Research, 204(3), $485-495$.

Borgonovo, E., Aliee, H., Glass, M., \& Teich, J. (2016). A new time-independent reliability importance measure. European Journal of Operational Research, 254, 427-442.

Brockman, P., \& Turtle, H. J. (2003). A barrier option framework for corporate security valuation. Journal of Financial Economics, 67(3), 511-529.

Cerqueti, R., \& Spizzichino, F. (2015). Signatures of systems with non-exchangeable lifetimes: some implications in the analysis of financial risk. In C. Kitsos, T. A. Oliveira, A. Rigas, \& S. Gulati (Eds.), Springer Proceedings in Mathematics \& Statistics, 136 (pp. 361-376). Springer.

Chen, W., Kritzman, M., \& Turkington, D. (2015). Alternative currency hedging strategies with known covariances. Journal of Investment Management, 13(2), 6-24. 
Dembinska, A. (2018). On reliability analysis of $k$-out-of- $n$ systems consisting of heterogeneous components with discrete lifetimes. IEEE Transactions on Reliability, 67(3), 1071-1083.

Eryilmaz, S. (2011). Dynamic behavior of k-out-of-n: G systems. Operations Research Letters, 39, $155-159$.

Eryilmaz, S. (2012). On the mean residual life of a k-out-of-n: G system with a single cold standby component. European Journal of Operational Research, 222, 273-277.

Eryilmaz, S. (2013). On reliability of a k-out-of-n system equipped with a single warm standby component. IEEE Transactions on Reliability, 62, 499-503.

Eryilmaz, S. (2016). Computing Barlow-Proschan importance in combined systems. IEEE Transactions on Reliability, 65, 159-163.

Eryilmaz, S. (2018). The number of failed components in a k-out-of-n system consisting of multiple types of components. Reliability Engineering \& System Safety, 175, 246-250.

Esary, J. D., \& Proschan, F. (1963). Coherent structures of non-identical components. Technometrics, 5(2), 191-209.

Faghih-Roohi, S., Xie, M., Ng, K. M., \& Yam, R. C. (2014). Dynamic availability assessment and optimal component design of multi-state weighted k-out-of-n systems. Reliability Engineering \& System Safety, $123,57-62$.

Franko, C., \& Tutuncu, G. Y. (2016). Signature based reliability analysis of repairable weighted k-out-of-n: G systems. IEEE Transactions on Reliability, 65(2), 843-850.

Freixas, J., \& Puente, M. A. (2009). Consecutive expansions of k-out-of-n systems. Operations Research Letters, 37, 438-442.

Gao, J., Yao, K., Zhou, J., \& Ke, H. (2018). Reliability analysis of uncertain weighted $k$-Out-of- $n$ systems. IEEE Transactions on Fuzzy Systems, 26(5), 2663-2671.

Gertsbakh, I. B., \& Shpungin, Y. (2010). Models of Network Reliability. Boca Raton: CRC Press.

Gurler, S., \& Bairamov, I. (2009). Parallel and k-out-of-n: G systems with nonidentical components and their mean residual life functions. Applied Mathematical Modeling, 33, 1116-1125.

Hobson, D., Laurence, P., \& Wang, T. H. (2005). Static-arbitrage optimal subreplicating strategies for basket options. Insurance: Mathematics and Economics, 37(3), 553-572.

Hull, J. C. (2006). Options, Futures, and Other Derivatives (6th ed.). Upper Saddle River, N.J.: Prentice Hall.

Kim, J., Kim, J., Yoo, H. J., \& Kim, B. (2015). Pricing external barrier options in a regime-switching model. Journal of Economic Dynamics and Control, 53, 123-143.

Kochar, S., Mukerjee, H., \& Samaniego, F. J. (1999). The signature of a coherent system and its application to comparisons among systems. Naval Research Logistics, 46(5), 507-523.

Li, W., \& Zuo, M. J. (2008). Reliability evaluation of multi-state weighted k-out-of-n systems. Reliability Engineering \& System Safety, 93(1), 160-167.

Lin, X. S. (1998). Double barrier hitting time distributions with applications to exotic options. Insurance: Mathematics and Economics, 23(1), 45-58.

Marichal, J.-L., \& Mathonet, P. (2011). Extensions of system signatures to dependent lifetimes: Explicit expressions and interpretations. Journal of Multivariate Analysis, 102(5), 931-936.

Marichal, J.-L., Mathonet, P., \& Spizzichino, F. (2015). On modular decompositions of system signatures. Journal of Multivariate Analysis, 134, 19-32.

McNeil, A. J., Frey, R., \& Embrechts, P. (2015). Quantitative risk management: Concepts, techniques and tools. Princeton: Princeton University Press.

Navarro, J., Spizzichino, F., \& Balakrishnan, N. (2010). Applications of average and projected systems to the study of coherent systems. Journal of Multivariate Analysis, 101(6), 1471-1482.

Navarro, J., del Aguila, Y., Sordo, M. A., \& Suarez Llorens, A. (2013). Stochastic ordering properties for systems with dependent identically distributed components. Applied Stochastic Models in Business and Industry, 29, 264-278.

Navarro, J., Pellerey, F., \& Di Crescenzo, A. (2015). Orderings of coherent systems with randomized dependent components. European Journal of Operational Research, 240, 127-139.

Navarro, J., Ruiz, J. M., \& Sandoval, C. J. (2005). A note on comparisons among coherent systems with dependent components using signatures. Statistics and Probability Letters, 27, 179-185.

Parsa, M., Di Crescenzo, A., \& Jabbari, H. (2018). Analysis of reliability systems via Gini-type index. European Journal of Operational Research, 264, 340-353.

Peña, J., Vera, J. C., \& Zuluaga, L. F. (2012). Computing arbitrage upper bounds on basket options in the presence of bid-ask spreads. European Journal of Operational Research, 222(2), 369-376.

Petchrompo, S., Li, H., Erguido, A., Riches, C., \& Parlikad, A. K. (2020). A value-based approach to optimising long-term maintenance plans for a multi-asset k-out-of-N system. Reliability Engineering \& System Safety, 106924,.

Samaniego, F. J. (1985). On closure of the IFR class under formation of coherent systems. IEEE Transactions on Reliability, R34, 60-72. 
Samaniego, F.J., System signatures and their applications in engineering reliability. International Series in Operations Research \& Management Science, 110. New York: Springer, 2007.

Sesana, D., Marazzina, D., \& Fusai, G. (2014). Pricing exotic derivatives exploiting structure. European Journal of Operational Research, 236(1), 369-381.

Shaked, M., \& Tong, Y. L. (1985). Some partial orderings of exchangeable random variables by positive dependence. Journal of Multivariate Analysis, 17(3), 333-349.

Sheng, Y., \& Ke, H. (2020). Reliability evaluation of uncertain k-out-of-n systems with multiple states. Reliability Engineering \& System Safety, 195, 106696.

Spizzichino, F. Subjective probability models for lifetimes. Boca Raton, FL: Chapman \& Hall/CRC. ISBN: 1-58488-060-0, 2001.

Spizzichino, F., The role of signature and symmetrization for systems with non-exchangeable components. In: Advances in Mathematical Modeling for Reliability, Amsterdam: IOS, 2008, 138-148.

Sun, C., Yang, J.-Y., \& Li, S.-H. (2007). On barrier option pricing in binomial market with transaction costs. Applied Mathematics and Computation, 189(2), 1505-1516.

Wang, Y., Li, L., Huang, S., \& Chang, Q. (2012). Reliability and covariance estimation of weighted k-out-of-n multi-state systems. European Journal of Operational Research, 221(1), 138-147.

Wu, J. S., \& Chen, R. J. (1994). An algorithm for computing the reliability of weighted k-out-of-n system. IEEE Transactions on Reliability, 43, 327-328.

Wu, S., \& Coolen, F. P. A. (2013). A cost-based importance measure for system components: An extension of the Birnbaum importance. European Journal of Operational Research, 225, 189-195.

Xu, G., \& Zheng, H. (2010). Basket options valuation for a local volatility jump-diffusion model with the asymptotic expansion method. Insurance: Mathematics and Economics, 47(3), 415-422.

Yamamoto, H., Akiba, T., Nagatsuka, H., \& Moriyama, Y. (2008). Recursive algorithm for the reliability of a connected-(1, 2)-or-(2, 1)-out-of-(m, n): F lattice system. European Journal of Operational Research, $188(3), 854-864$.

Zhang, N., Fouladirad, M., \& Barros, A. (2019). Reliability-based measures and prognostic analysis of a $K$-outof- $N$ system in a random environment. European Journal of Operational Research, 272(3), 1120-1131.

Zhang, P. G. (1997). Exotic Options. Singapore: World Scientific Publishing Co.

Zhang, Y. (2021). Reliability analysis of randomly weighted k-out-of-n systems with heterogeneous components. Reliability Engineering \& System Safety, 205, 101784.

Publisher's Note Springer Nature remains neutral with regard to jurisdictional claims in published maps and institutional affiliations. 